Meta

Journal des traducteurs

Translators' Journal

\title{
Terminologie pharmaceutique
}

\section{Suzanne Desjardins}

Volume 12, numéro 4, décembre 1967

Aspects de la terminologie

URI : https://id.erudit.org/iderudit/002549ar

DOI : https://doi.org/10.7202/002549ar

Aller au sommaire du numéro

Éditeur(s)

Les Presses de l'Université de Montréal

ISSN

0026-0452 (imprimé)

1492-1421 (numérique)

Découvrir la revue

Citer cet article

Desjardins, S. (1967). Terminologie pharmaceutique. Meta, 12(4), 130-133.

https://doi.org/10.7202/002549ar

Ce document est protégé par la loi sur le droit d'auteur. L'utilisation des services d'Érudit (y compris la reproduction) est assujettie à sa politique d'utilisation que vous pouvez consulter en ligne.

https://apropos.erudit.org/fr/usagers/politique-dutilisation/
Cet article est diffusé et préservé par Érudit.

Érudit est un consortium interuniversitaire sans but lucratif composé de l'Université de Montréal, l'Université Laval et l'Université du Québec à Montréal. Il a pour mission la promotion et la valorisation de la recherche. https://www.erudit.org/fr/ 


\section{TERMINOLOGIE PHARMACEUTIQUE.}

Le Comité d'étude des termes de médecine a reçu du corps médical un appui unanime dans son œuvre d'épuration de la langue scientifique. Les pharmaciens, à leur tour, désirent participer à la réforme du français. Le Comité a donc chargé une commission ad hoc d'étudier les termes pharmaceutiques et de proposer un vocabulaire des expressions les plus courantes. La Commission se compose d'un pharmacien, de deux médecins qui exercent leur prof́ession dans l'industrie pharmaceutique et de trois traducteurs spécialisés dans les textes médicaux. Le docteur Georges Desrosiers, président du Comité d'étude des termes de médecine, en dirige les travaux.

Nous avons adopté la méthode de travail du Comité d'étude des termes de médecine, qui est également celle du Comité d'étude des termes techniques français et du Comité de linguistique de Radio-Canada. Les membres proposent des expressions à étudier qui font chacune l'objet d'une fiche. Tantôt il s'agit de fiches correctives destinées à déraciner des emplois et usages fautifs, tantôt de fiches de complément tendant à combler les lacunes de la langue, tantôt enfin de fiches de traduction. Dans ce dernier cas, chaque fiche comporte la définition de l'expression anglaise, le terme français équivalent et, à titre de justification, un exemple tiré d'un bon auteur français. Lorsqu'une trentaine de fiches ont été discutées, elles sont soumises à l'examen de l'Office de la langue française du Québec. À la lumière des recommandations de cet organisme, la Commission revise chaque fiche et procède au besoin à de nouvelles études. 
Dans l'industrie pharmaceutique, nous devons tenir compte non seulement de la langue scientifique, mais aussi d'une certaine forme commerciale puisque nous avons des produits à vendre. Le rédacteur doit savoir doser avec soin l'otalgie et le mal d'oreille, l'angine et le mal de gorge, la sapidité et le goût agréable selon qu'il s'adresse aux médecins ou au public.

À ces subtilités de la langue s'ajoute au Canada l'influence de l'anglais sur la terminologie pharmaceutique. Par exemple, la loi fédérale, rédigée en anglais, distingue deux catégories de médicaments: les ethical drugs, d'une part, et les patent medicines ou proprietary drugs, de l'autre. La mise au point de traductions adéquates pour ces expressions a nécessité de longues heures de recherche et d'étude, tant ces deux expressions anglaises sont peu explicites.

La locution ethical drugs désigne, selon la Direction des aliments et drogues du Ministère fédéral de la Santé, les médicaments qui, légalement, ne peuvent être vendus que sur ordonnance. Ils sont ainsi nommés parce que la publicité qui les concerne s'adresse exclusivement aux corps professionnels, c'est-à-dire aux médecins et aux pharmaciens.

Les locutions proprietary drug ou patent medicine désignent une préparation pharmaceutique ou un médicament artificiel fabriqué pour usage interne ou externe et dont le nom, la composition ou la définition ne se trouvent dans aucune pharmacopée ni aucun Codex ou formulaire adoptés par une association pharmaceutique dûment reconnue par l'État et dont la véritable formule ou la liste des ingrédients médicinaux qui le composent n'apparaissent pas sur le contenant.

D'ailleurs la cloison entre ces deux catégories de médicaments n'est pas entièrement étanche: certaines ethical drugs ne sont pas vendues sur ordonnance, et les patent medicines ne sont pas toutes mises en vente libre. En anglais, le mot ethical implique que l'utilisation de ces médicaments exige l'intervention des corps médical et paramédical, donc d'organismes régis par un code déontologique (ethical code). Pourtant certaines maisons qui fabriquent de ces substances en fabriquent d'autres aussi. Il fallait en outre tenir compte de la différence établie par la loi entre un brevet et une marque déposée. Après trois ou quatre séances, la Commission des termes pharmaceutiques s'est ralliée à la solution suivante: l'expression spécialités pharmaceutiques rendrait ethical drugs, tandis qu'on réserverait l'appellation médicaments brevetés aux proprietary drugs. Or, après plusieurs semaines de retard, nous arrive d'Ottawa le texte français de la loi concernant les substances brevetées. Le texte est intitulé: Loi concernant les spécialités pharmaceutiques ou médicaments brevetés. Le problème reste donc entier: le traducteur a fait de purs synonymes des deux expressions qui nous avaient permis de distinguer les deux catégories de médicaments en question. Heureusement pour le traducteur, seul le texte anglais a force de loi. Il lui est donc loisible d'adopter sans danger les deux expressions proposées. D'ailleurs, il appert que la loi abandonnera bientôt ces distinctions qui ne vaudraient plus que dans l'industrie.

Une autre expression âprement discutée touche au domaine des contraceptifs. Il s'agit de la traduction du terme sequential, appliqué à certaines formes de thérapeutique anovulatoire comportant deux phases successives. Or, nous relevons dans 
la Presse médicale ${ }^{1}$ une définition de la « thérapeutique hormonale séquentielle qui consiste à réaliser un cycle artificiel proche du cycle normal en associant cestrogènes et progestinogènes selon les modalités suivantes... ». De son côté, le Comité des termes de médecine avait déjà noté que le terme séquentiel est utilisé en statistique de même qu'en télévision où il s'applique à un procédé de transmission d'éléments d'image acheminés en succession. L'usage de ce terme se généralise en France pour désigner un traitement comportant des phases successives. Il semblait donc possible d'accepter le terme séquentiel.

Pourtant, une donnée nouvelle est venue compliquer la situation. Dans sa livraison du 30 avril 1966, la Presse médicale publie un article intitulé: «Effets physiologiques des œstrogènes sur la fonction ovarienne ${ }^{2}$, portant en sous-titre: "Étude de la suppression de l'ovulation par la thérapeutique «diphasique». Un astérisque renvoie à la note suivante: «Dite « séquentielle», mais ce terme est pour l'instant un barbarisme ». La question a donc été remise à l'étude.

Notre Commission en a conclu qu'il existe une nuance entre les deux idées: on peut concevoir un traitement à plusieurs phases qui ne seraient pas strictement consécutives. Les adjectifs diphasique, triphasique, etc., pourraient alors trouver place à côté du «barbare » séquentiel, du moins tant que les spécialistes en linguistique ne se seront pas prononcés.

Une autre expression, quoique moins nouvelle, a néanmoins été l'objet d'une discussion prolongée. Il s'agit de l'adjectif extemporané qui s'appliquait tout d'abord à une substance préparée sur le champ (du latin ex tempore). Certains membres de notre Commission ne voyaient pas d'un bon œil l'extension de cet adjectif au sens de «prêt à l'emploi ». Heureusement nous avons relevé sous la plume d'un distingué linguiste européen, H. Van Hoof, chroniqueur linguistique de la Presse médicale, la phrase suivante, qui nous a paru une justification suffisante: « Si l'expression «prêt à l'usage » est correcte, elle n'en est pas moins très peu pharmaceutique et l'on doit lui préférer le terme extemporané.»

Le terme américain disposable a également retenu l'attention non seulement de la Commission des termes pharmaceutiques mais aussi du Comité d'étude des termes de médecine. L'équipe Clair-dire, dirigée par le docteur Eyraud, et vouée en France à l'épuration de la langue médicale, a retenu pour traduire cette réalité nouvelle l'expression jetable. Notre Commission en revanche défend uniservice.

Le Comité des termes de médecine a conclu à l'utilité des deux expressions: uniservice s'appliquant aux objets qui ne peuvent servir qu'une fois, telle la literie de papier; jetable s'appliquant aux objets qui peuvent être récupérés, mais sans profit. Il existe, par exemple, des seringues dites disposable ou jetables qu'on récupère pourtant dans certains hôpitaux de Montréal à l'intention des pays sousdéveloppés où, grâce à une main-d'œuvre abondante et bon marché, il est profitable de faire subir à ces instruments des usages répétés. Ici apparaît la tendance, si fréquente en français, à utiliser plusieurs termes spécifiques là où l'anglais se contente d'un seul terme générique.

1. Vol. $74, \mathrm{n}^{\circ} 1,1^{\mathrm{er}}$ janvier 1966, p. 14

2. Vol. $74, n^{\circ} 22$, p. 1129. 
Parmi les termes purement publicitaires, notre Commission a décidé, à l'instar du Comité de linguistique de Radio-Canada, d'écarter le mot «exhibit» au profit de stand, déjà reçu par l'usage. Les visiteurs médicaux érigeront à l'avenir dans les hôpitaux ou dans les salles de congrès des stands où ils auront des pièces d'exposition scientifiques ou commerciales selon le cas. De plus, lorsqu'ils se présenteront au cabinet de consultation du médecin, ils apporteront non plus une "aide visuelle » (de l'anglais visual aid) mais bien une pièce de démonstration comportant des éléments graphiques ou visuels de propagande ou de publicité. L'industrie pharmaceutique doit aussi lutter contre les interférences linguistiques résultant de la prépondérance de la langue anglaise dans le monde des affaires. Nous luttons avec acharnement contre des affections toutes aussi graves les unes que les autres: néologismes ambigus ou contrefaits, xénismes anglo-saxons, calques, faux amis, indigence ou pléthore lexicales, impropriétés, et les nombreux épiphénomènes qui entraînent sur le plan linguistique des effets toxiques (contresens, faux sens, glissements sémantiques abusifs).

Le succès de cette lutte dépendra de l'intérêt que les usagers témoigneront à la correction du langage. Les comités ou commissions proposent, mais les usagers disposent.

SUZANne DesJaRdins 